\title{
Surface/strain energy balance controlling preferred orientation in CdZnO films
}

T. C. Zhang and, and A. Yu. Kuznetsov

Citation: Journal of Applied Physics 110, 053512 (2011); doi: 10.1063/1.3632071

View online: http://dx.doi.org/10.1063/1.3632071

View Table of Contents: http://aip.scitation.org/toc/jap/110/5

Published by the American Institute of Physics

\section{AlP | Journal of A1P Aplied Physics}

Save your money for your research.

It's now FREE to publish with us no page, color or publication charges apply.
Publish your research in the

Joumal of Applied Physics

to claim your place in applied

physics history. 


\title{
Surface/strain energy balance controlling preferred orientation in $\mathrm{CdZnO}$ films
}

\author{
T. C. Zhang and A. Yu. Kuznetsov \\ Department of Physics/Centre for Materials Science and Nanotechnology, University of Oslo, Blindern, \\ Oslo N-0316, Norway
}

(Received 17 June 2011; accepted 30 July 2011; published online 9 September 2011)

\begin{abstract}
Single-phase rock-salt $\mathrm{CdZnO}$ films were synthesized on r-plane sapphire substrates by metal-organic chemical vapor deposition. Evolutions in growth orientations were investigated in these films as a function of $\mathrm{Zn}$ content and film thickness. The preferred orientation is found to black (thick) and red (thin) lines depend on the balance between the surface and strain energy accumulated in the films in accordance with the so-called overall energy model. Specifically, (100) orientation dominates below a critical Zn content and/or a critical film thickness, otherwise (111)-oriented grains nucleate on top of the (100) planes. (C) 2011 American Institute of Physics. [doi:10.1063/1.3632071]
\end{abstract}

\section{INTRODUCTION}

In recent years, transparent conducting oxides (TCOs) have attracted much attention as critical components in various optoelectronic devices, specifically flat panel displays, solar cells, etc. For example, tin-doped indium oxide is used as a typical TCO in spite of several disadvantages (high cost of indium, relatively low conductivity, etc.), which are foreseen to $\operatorname{limit}^{1}$ its application calling for alternative TCO research. Cadmium oxide $(\mathrm{CdO})$ is an n-type semiconductor and regarded as a promising TCO material, too, owing to its reasonable transmittance and low resistivity. Even though the $\sim 2.5 \mathrm{eV}$ direct bandgap of $\mathrm{CdO}$ (note different reports quote direct bandgap of $\mathrm{CdO}$ in the range of $2.25-2.9 \mathrm{eV}$ ) determines its relatively limited transparency in shorter wavelength regions, the case may be improved by alloying it with $\mathrm{ZnO}$ resulting in broadening of the bandgap. ${ }^{2}$ Moreover, an interesting application for $\mathrm{CdO}$ (and $\mathrm{CdZnO}$ ) may be to use it as a light-absorbing semiconductor in solar cells. ${ }^{3-5}$ Various deposition methods were tested to manufacture $\mathrm{CdO}$ films, including spray pyrolysis, ${ }^{6}$ sputtering, ${ }^{7}$ solgel, ${ }^{8}$ pulsed laser deposition, ${ }^{9}$ and metal-organic chemical vapor deposition (MOCVD). ${ }^{10-12}$ A significant effort has been made to understand the relationship between the deposition conditions and the properties of $\mathrm{CdO}$ targeting improvement in the structural quality ${ }^{9}$ and device performance. ${ }^{13}$ Nevertheless, there are several issues remaining unresolved. For example, an evolution in preferred growth orientations was observed in $\mathrm{CdO}$ films, ${ }^{4,10}$ but its nature has not been clearly explained. The complexity of the problem will obviously increase in $\mathrm{CdZnO}$ because of the competition between the rock-salt (RS) and wurtzite (W) symmetries originating from the binary components. In its turn, the investigation of the preferred grain orientation enhances the understanding of fundamental mechanisms controlling film growth and thus helps to master high-quality $\mathrm{CdZnO}$ alloys. In this paper, the evolution of the preferred growth orientation was investigated in $\mathrm{RS}-\mathrm{CdZnO}$ films as a function of $\mathrm{Zn}$ content and film thickness. The results are explained in terms of the surface/strain energy balance determining the preferred orientation in the films.

\section{EXPERIMENTAL}

$\mathrm{CdZnO}$ films were synthesized on r-plane sapphire substrates by MOCVD. More detailed descriptions of the reactor, precursor delivery, substrate pretreatments, etc., can be found elsewhere. ${ }^{14}$ In short, DMCd, DEZn, and t-BuOH were employed as $\mathrm{Cd}, \mathrm{Zn}$, and $\mathrm{O}$ sources carried by highpurity $\mathrm{N}_{2}$ gas. Two sets of samples were prepared. For the first set (Set A), $\mathrm{Cd}_{1-x} \mathrm{Zn}_{x} \mathrm{O}$ films with different $\mathrm{Zn}$ contents $x=0,0.07,0.11,0.20,0.30,0.49$, and 0.56 were deposited by varying $\mathrm{DEZn} / \mathrm{DMCd}$ ratio keeping the film thickness constant at $\sim 800 \mathrm{~nm}$. These samples are labeled as ZXX, where XX denotes Zn content, e.g., Z00 and Z20 means pure $\mathrm{CdO}$ and $\mathrm{Cd}_{0.8} \mathrm{Zn}_{0.2} \mathrm{O}$, respectively. The elemental chemical analysis of the samples was performed by Rutherford backscattering spectrometry (RBS) and time-of-flight elastic recoil detection analysis (ToF-ERDA) so that Fig. 1 plots the chemical composition as a function of precursor molar ratios used during synthesis of Set A samples. The use of RBS/ ToF-ERDA combination ensured good sensitivity to both heavy $(\mathrm{Zn}, \mathrm{Cd})$ and light $(\mathrm{O})$ elements. For the second set of samples (Set $\mathrm{B}$ ), $\mathrm{Cd}_{0.8} \mathrm{Zn}_{0.2} \mathrm{O}$ alloys (corresponding to the cross symbol in Fig. 1) were synthesized in the form of films having different thickness $(\sim 15,70,150,200,400$, and $800 \mathrm{~nm}$ ) and correspondingly labeled as T15, T70, etc.

Structural properties of the samples were investigated by x-ray diffraction (XRD) employing a predominant $\mathrm{Cu} \mathrm{K}_{\alpha 1}$ radiation collected into a scintillation detector. Note, that $\mathrm{K}_{\beta}$-related diffractions may still be detected in the XRD configuration used, even though with much lower intensity (which was nevertheless used as an advantage in peak identification). The surface morphology and, specifically, surface plane geometry were investigated by scanning electron microscopy (SEM). 


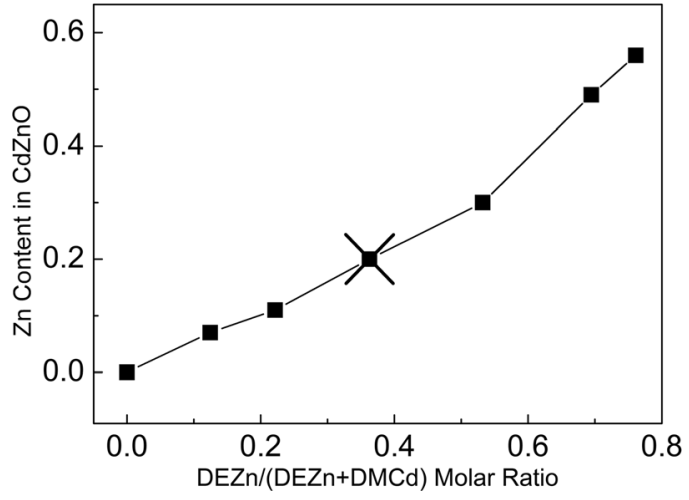

FIG. 1. $\mathrm{Zn}$ content in $\mathrm{CdZnO}$ films as a function of molar flow ratio used during synthesis. Squares label $\sim 800 \mathrm{~nm}$ films exhibiting different compositions (Set A), and the cross labels the synthesizing condition/composition of the samples exhibiting thickness variations (Set B).

\section{RESULTS AND DISCUSSION}

Figure 2 shows typical XRD $2 \theta$-scans taken from (a) Set A, i.e., $\mathrm{Cd}_{1-x} \mathrm{Zn}_{x} \mathrm{O}$ samples exhibiting $x$ variations, and (b) Set $\mathrm{B}$, i.e., $\mathrm{Cd}_{0.8} \mathrm{Zn}_{0.2} \mathrm{O}$ samples having different thickness. The XRD pattern confirms cubic single-phase behavior of the samples as long as W-related peaks (e.g., associated with $\mathrm{W}-\mathrm{ZnO}$ ) were not detected. Further, sample $\mathrm{Z00}$ (pure $\mathrm{CdO}$ in Fig. 2(a)) demonstrates well-oriented film as confirmed by (200)-related diffraction detected by both $\mathrm{K}_{\alpha 1}$ (at $\sim 38.3^{\circ}$ ) and $\mathrm{K}_{\beta}\left(\right.$ at $\left.\sim 34.5^{\circ}\right)$ radiations. The fact that the rest of the samples in Fig. 2(a) demonstrate a strong diffraction at $\sim 38.3^{\circ}$ is a clear indication that $\mathrm{CdZnO}$ adapts (100) as its preferred growth orientation on r-sapphire, too, consistent with the literature. ${ }^{10}$ The position of this (200) peak clearly shifts toward high angles initially (samples Z07 and Z11), presumably because of the shrinking of the lattice constant resulting from the incorporation of $\mathrm{Zn}$ atoms-having smaller ionic radius $(0.74 \AA)$ (Ref. 15) as compared to that of Cd (0.95 ̊) (Ref. 15) —on equivalent sites. Interestingly, for higher $\mathrm{Zn}$ content samples, the (200) peak moves back to

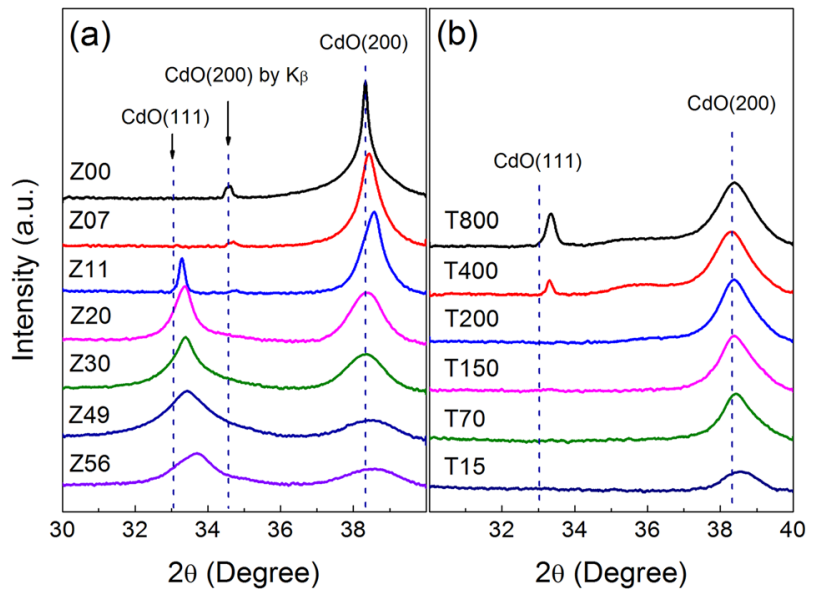

FIG. 2. (Color online) Typical $2 \theta$ XRD patterns taken from (a) samples in Set A with different $\mathrm{Zn}$ content, and (b) samples in Set B with different film thickness, respectively. the lower angle positions in spite of a gradual increase of $\mathrm{Zn}$ content. This behavior may be consistently understood considering the amount of material present in the films in the form of (100)-oriented grains as will be discussed later. Concurrently, RS-(111)-related diffraction obviously rises up in $\mathrm{Z} 11$, and this peak shifts gradually toward high angles as a function of $\mathrm{Zn}$ content. Interestingly, a weak indication of RS-(111) plane may be traced even in Z07, as will be confirmed by SEM studies shown below. Originally, there is a clear trend of complete (100) orientation to mix with (111) orientation as a function of increasing $\mathrm{Zn}$ content (see Fig. 2(a)).

Further, investigating Fig. 2(b), one may immediately observe an evolution in the preferential grain orientation in the films as a function of thickness. Indeed, there is a single RS-(200) peak observed in T15-T200, whereas the characteristic RS-(111) reflection emerges in T400 and develops in T800, confirming that the growth orientation is affected by the thickness variations. Similarly to that in Fig. 2(a), some peaks in Fig. 2(b) exhibit shifts and/or broadening with respect to the positions characteristic for pure $\mathrm{CdO}$. Overall, the epitaxial relationship and corresponding tensile strain accumulation because of the in-plane lattice mismatch between $\mathrm{CdO}$ and $\mathrm{r}-\mathrm{Al}_{2} \mathrm{O}_{3}$ has been reported in the literature. ${ }^{16}$ The shrinking of the lattice constant of $\mathrm{CdZnO}$ parallel to the surface normal under in-plane tensile strain is a usual Poisson effect. Naturally, as the film thickness increases and the coherence with the substrate weakens, the characteristic XRD peaks shift toward similar positions (see Fig. 2(b)). Z20 and T800 samples were processed using nominally the same growth parameters (see Fig. 1) but from different batches. Fundamentally, these samples show very similar XRD patterns but slightly different (111)/(200) peak intensity ratio.

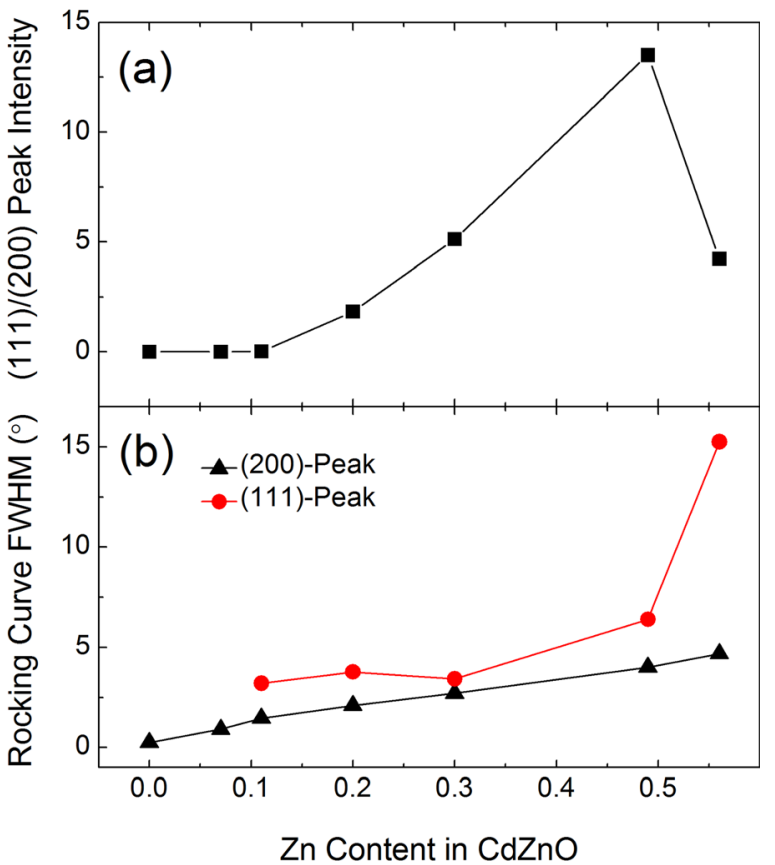

FIG. 3. (Color online) (a) XRD (111)/(200) peak intensity ratios, and (b) FWHM of rocking curves as a function of $\mathrm{Zn}$ content. 
Figure 3 plots (a) (111)/(200) peak intensity ratios, and (b) full width at half maximum (FWHM) of (111) and (200)related rocking curves for Set A as a function of $\mathrm{Zn}$ content. In the interpretation of Fig. 3(a), we take into account that the Bragg intensity is proportional to the volume of the grains having the corresponding orientations. In these terms, the shape of the (111)/(200) intensity ratio versus $\mathrm{Zn}$ content dependence (see Fig. 3(a)) suggests that the volume of the (111)-grains increases gradually in samples Z00-Z49. A similar tendency holds for the broadening of rocking curves (Fig. 3(b)), which is readily translated into degree of deviation from a preferred growth orientation. Note, the reflection intensity decreases considerably and appears to be less dependent on the grain-volume relationship in Z56, likely because of a more significant disorientation of the (111) grains compared to that of (100) because of a different localization of the corresponding grains. A scenario for the grain orientation evolution is suggested as a result of the discussion of the surface morphology below.

Surface morphologies of several selected samples are depicted in Fig. 4, so that panels (a) and (b) illustrate the

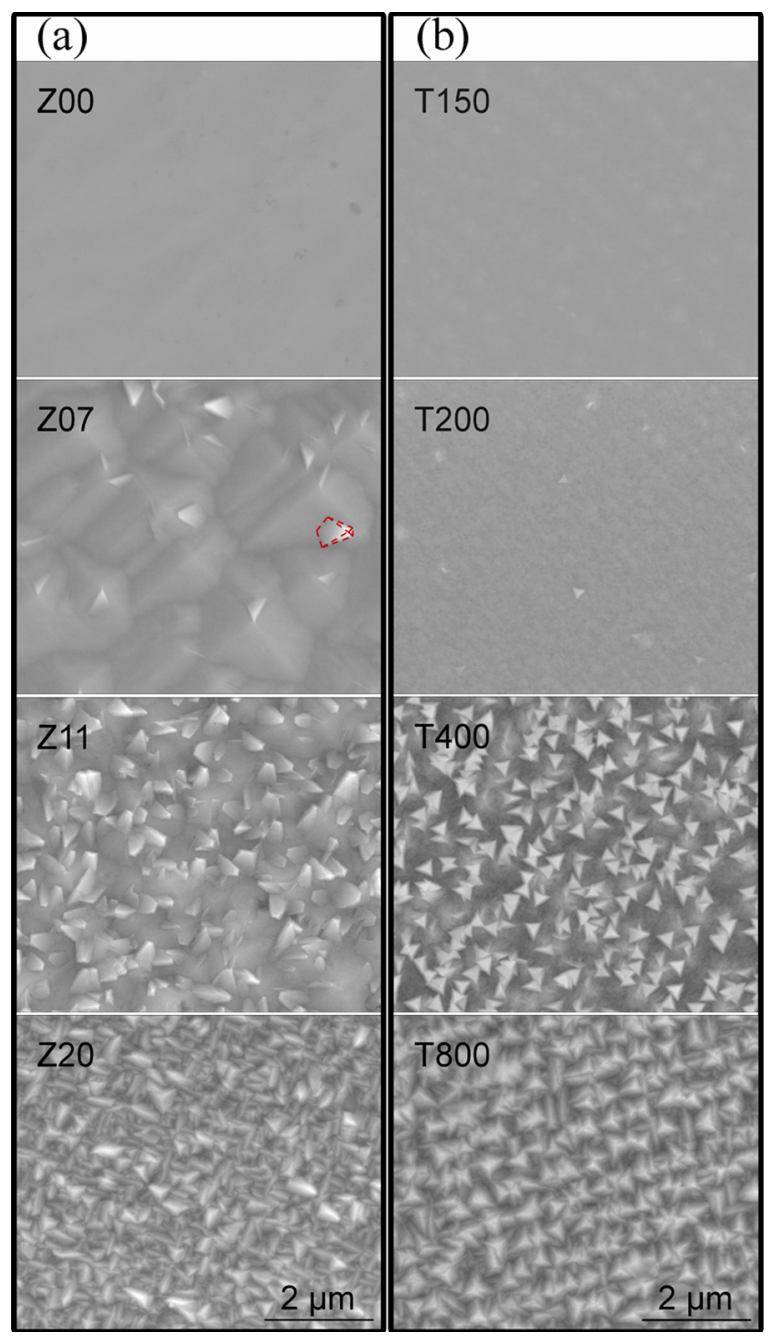

FIG. 4. (Color online) SEM images of (a) $\mathrm{Cd}_{1-x} \mathrm{Zn}_{x} \mathrm{O}$ samples with variable $\mathrm{Zn}$ content, and (b) $\mathrm{Cd}_{0.8} \mathrm{Zn}_{0.2} \mathrm{O}$ samples with different film thickness. The dashed-line frame indicates the typical "pyramid-like" features developing in the samples. surface morphology evolution as a function of $\mathrm{Zn}$ content and film thickness, respectively. Starting with Fig. 4(a), the pure $\mathrm{CdO}$ film (Z00 sample) exhibits a smooth surface. As soon as a sufficient amount of $\mathrm{Zn}$ is incorporated into the film (e.g., in sample Z07), a pyramid-like surface morphology develops (the pattern is visualized by a dashed-line frame in Fig. 4(a)). The nucleation rate for such pyramids increases with increasing $\mathrm{Zn}$ content, so that the pyramids eventually merge together and cover the entire surface (see the evolution in Fig. 4(a)). Similar trends are observed in the morphology evolutions as a function of the film thickness in Fig. 4(b). Indeed, a smooth surface is observed for T15-T150 samples (not shown for T15 and T70) but it evolves to a surface densely covered by a large number of pyramids in T400. Taking into account the XRD results/analysis in Figs. 2 and 3, indicating appearance of RS-(111) reflections for alloys with composition $x \geq 0.11$ (or thickness $\geq 400 \mathrm{~nm}$ ), and also the increasing intensity ratio of (111) over (200) reflections with increasing the density of the corresponding grains, we conclude that the pyramid-like features are associated with RS-(111) $\mathrm{CdZnO}$ grains whereas the flat surface naturally corresponds to the (100) orientation. Note that the observation of pyramid-like features in Z07 in Fig. 4(a) indicates that (111) orientation has emerged already at low $\mathrm{Zn}$ content samples; however, the corresponding volume of these (111)-gains was insufficient to make it well resolved in our XRD measurements in Fig. 2. We assume that the preferred film orientation starts to change in $\mathrm{CdZnO}$ when $\mathrm{Zn}$ content is $\geq 7 \%$. Similarly to that in Z07, the (111) orientation starts to develop in T200 when employing thickness variations in Set B. Importantly, similar morphology evolutions as a function of $\mathrm{Zn}$ content and/or film thickness imply that the conversion of preferred orientation is governed by a mechanism having a similar nature.

At this point, we would like to recapitulate the angular positions of (200) peaks in Fig. 2(a), specifically for the high $\mathrm{Zn}$ content samples. The fact that the lattice parameter, e.g., in Z20, considering (100)-oriented grains, is not decreasing (in spite of incorporating more of smaller size $\mathrm{Zn}$ atoms comparing to that in Z07), is consistent with shrinking of the relative volume occupied by these (100)-oriented grains (see Fig. 3(a)) localized in Z20 in the vicinity of the substrate/ film interface only, because of the change in the preferential growth orientation in the rest of the film (see Fig. 4(a)). Naturally, the substrate misfit-related strain influences a thinner (100)-oriented part of the films in a bigger proportion compared to that in the films consisting predominantly of (100)oriented grains, e.g., in Z07.

Generally speaking, the preferred growth orientation is determined predominantly by the substrate surface orientation providing an optimal match for the epitaxial growth. (The difference in thermal expansion coefficients between substrate and film can introduce strain as well, which could influence the orientation of the film, but we take it out of consideration assuming it to be a minor systematic error as all of our samples were grown on the same substrates.) As discussed above, the lowest lattice mismatch between RS$\mathrm{CdO}$ and r-plane $\mathrm{Al}_{2} \mathrm{O}_{3}$ substrate may be anticipated for (100) planes, whereas other preferential $\mathrm{CdO}$ orientations 


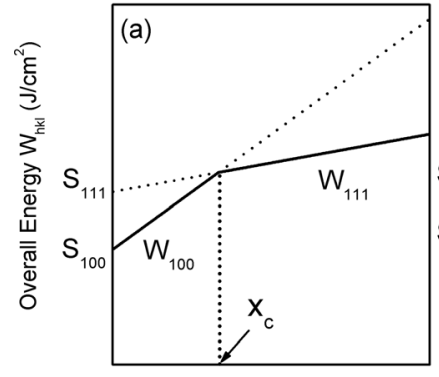

Zn Content

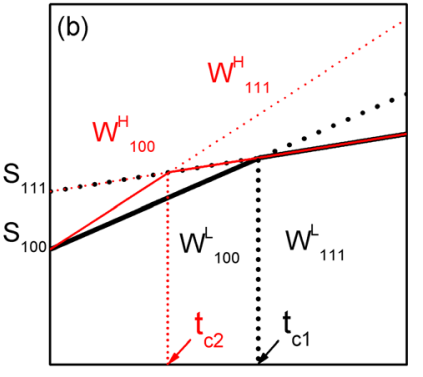

Film Thickness
FIG. 5. (Color online) Schematics of surface and strain energy contributions to the overall energy of $\mathrm{CdZnO}$ film as a function of (a) $\mathrm{Zn}$ content, and (b) film thickness. Black (thick) and red (thin) plots in (b) represent the overall energies from lower and higher $\mathrm{Zn}$ content samples.

can be obtained on other $\mathrm{Al}_{2} \mathrm{O}_{3}$ cuts. ${ }^{10}$ Having these facts in mind, any possible evolutions in the growth orientation are controlled by the balance between surface and strain energies accumulated in the films. ${ }^{17}$ Specifically, the change of the strain energy may be implied by the incorporation of foreign atoms acting as sources of stress regarded as an intrinsic stress generated during film growth. ${ }^{18}$ Note that the accumulation of the strain energy in $\mathrm{CdZnO}$ films might be a function of $\mathrm{Zn}$ incorporation, so that the originally optimal $\mathrm{CdO}$ (100) matching to $\mathrm{r}-\mathrm{Al}_{2} \mathrm{O}_{3}$ goes out of balance along with the strain accumulation. As a result, a less energydemanding surface orientation may be provoked. An estimate for the surface energy can be made considering the sublimation energy in relation to the number of broken bonds on a certain plane. Specifically, it is known that (100) and (111) planes are associated with the lowest and highest surface energies, respectively, for low index planes in rock-salt structure. In contrast, the strain energy accumulates more readily along (100) orientation compared to that along (111) because of the anisotropy of the elastic moduli. ${ }^{17,19,20}$ As a result, we may expect a balance between (100) and (111) orientation because of the evolution of strain and surface energies in $\mathrm{CdZnO}$ films.

Figure 5 shows a schematic plotting of the evolution of the overall energy $(\mathrm{W})$, defined as a sum of surface and strain energies, in a RS-CdZnO film, taking into account both $\mathrm{Zn}$ content and film thickness dependences as observed in Figs. 2-4. By definition, $S_{100}$ and $S_{111}$ label surface energies (in $\mathrm{Z} 00$ pure $\mathrm{CdO}$ sample) associated with (100) and (111) planes, respectively. Zn incorporation may influence the surface energy (CRC Handbook of Chemistry and Physics, 51st ed., F-158-F-159 (1970-1971)) resulting in the bending over of both curves in Fig. 5(a) without affecting our conclusion. In accordance with the literature, $S_{100}<S_{111 .}{ }^{20}$ Further, assuming $\mathrm{W}_{100}$ and $\mathrm{W}_{111}$ to be overall energies for films having corresponding orientations, we account for that in pure $\mathrm{CdO} \mathrm{W}_{100}<\mathrm{W}_{111}$. Considering first the $\mathrm{Zn}$ content dependence in Fig. 5(a), we use the fact that the slope for the $\mathrm{W}_{100}$ line is larger than that for $\mathrm{W}_{111}$ because of the corresponding relationship of the elastic moduli. ${ }^{17}$ As a result, when $\mathrm{Zn}$ content exceeds a critical value $\left(x_{\mathrm{c}}\right)$, which likely happens in Z07, the preferred orientation changes from (100) to (111) lowering the overall energy.
Concurrently, it is well known that the strain energy may be a function of the film thickness. Thus, it can be reasonably expected that the preferred growth orientation of the film changes from (100) to (111) when the film thickness exceeds a critical thickness (see Fig. 5(b)). Now, $S_{100}$ and $S_{111}$ represent the surface energies for (100) and (111) planes for a certain $\mathrm{Cd}_{1-x} \mathrm{Zn}_{x} \mathrm{O}$ composition. Accounting for the thickness variations, the overall energy increase is a consequence of the strain energy accumulation, again with a higher slope in $\mathrm{W}_{100}$ comparing to that in $\mathrm{W}_{111}$ because of the higher/lower strain occurring in differently oriented grains. Because the surface energy provides dominating contributions into $\mathrm{W}$ for thinner samples, the (100) orientation is observed in T15-T150. However, as the thickness exceeds a critical value $\left(t_{\mathrm{c}}\right)$, it becomes more favorable to switch to (111) orientation to minimize W (see T400 and T800 in Fig. 2(b)). For the Set B samples, $t_{\mathrm{c}}$ is $\sim 200 \mathrm{~nm}$. Note that Fig. 5(b) also illustrates a natural influence of different $\mathrm{Zn}$ contents on the $t_{\mathrm{c}}$ values. As seen in Fig. 5(b), black (thick) and red (thin) lines plot the overall energies in the films having low and high Zn contents, respectively. Indeed, lower $\mathrm{Zn}$ content is expected to lead to less strain accumulated in the film, resulting in a larger critical thickness $\left(t_{\mathrm{c} 1}\right)$ as confirmed by comparing Z07 and T200 samples in Fig. 4 exhibiting $t_{\mathrm{c}}$ values of $\sim 800$ and $\sim 200 \mathrm{~nm}$, respectively.

\section{CONCLUSIONS}

Single-phase rock-salt $\mathrm{CdZnO}$ films were synthesized on r-plane sapphire substrates by MOCVD. The evolution in the film growth orientation was systematically investigated as a function of $\mathrm{Zn} / \mathrm{Cd}$ stoichiometric ratios and the film thickness. It is found that the preferred growth orientation is determined by a balance between surface and strain energies accumulated in the films as interpreted in terms of the overall energy model. Specifically, (100) orientation dominates below a critical $\mathrm{Zn}$ content and/or a critical film thickness; otherwise, development of (111)-oriented grains are provoked on top of the (100) planes.

\section{ACKNOWLEDGMENTS}

Partial financial support provided by the Research Council of Norway via FRINAT "Understanding ZnO” project and the Nordic Energy Research Agency via N-INNER "SolarH2" project is gratefully acknowledged. Dr. Azarov is acknowledged for providing $\mathrm{Zn} / \mathrm{Cd}$ concentration calibrations.

${ }^{1}$ Y. Yang, S. Jin, J. E. Medvedeva, J. R. Ireland, A. W. Metz, J. Ni, M. C. Hersam, A. J. Freeman, and T. J. Marks, J. Am. Chem. Soc. 127, 8796 (2005).

${ }^{2}$ V. Venkatachalapathy, A. Galeckas, M. Trunk, T. C. Zhang, A. Azarov, and A. Yu. Kuznetsov, Phys. Rev. B 83, 125315 (2011).

${ }^{3}$ I. Shih, S. Jatar, C. H. Champness, and N. Liria, Solar Cells 7, 327 (1982).

${ }^{4}$ K. T. R. Reddy, C. Sravani, and R. W. Miles, J. Cryst. Growth $\mathbf{1 8 4} / \mathbf{1 8 5}$, 1031 (1998).

${ }^{5}$ V. Venkatachalapathy, A. Galeckas, R. Sellappan, D. Chakarov, and A. Yu. Kuznetsov, J. Cryst. Growth 315, 301 (2011).

${ }^{6}$ C. Sravani, P. S. Reddy, and P. J. Reddy, Mater. Lett. 12, 406 (1992).

${ }^{7}$ K. Gurumurugan, D. Mangalaraj, and S. K. Narayandass, J. Electron. Mater. 25, 765 (1996). 
${ }^{8}$ Y.-S. Choi, C.-G. Lee, and S. M. Cho, Thin Solid Films 289, 153 (1996). ${ }^{9}$ M. Yan, M. Lane, C. R. Kannewurf, and R. P. H. Chang, Appl. Phys. Lett. 78, 2342 (2001).

${ }^{10}$ J. Zuniga-Perez, C. Martinez-Tomas, and V. Munoz-Sanjose, Phys. Status Solidi C 2, 1233 (2005).

${ }^{11}$ A. W. Metz, J. R. Ireland, J. G. Zheng, R. P. S. M. Lobo, Y. Yang, J. Ni, C. L. Stern, V. P. Dravid, N. Bontemps, C. R. Kannewurf, K. R. Poeppelmeier, and T. J. Marks, J. Am. Chem. Soc. 126, 8477 (2004).

${ }^{12}$ D. M. Ellis and S. J. C. Irvine, J. Mater. Sci.: Mater. Electron. 15, 369 (2004).

${ }^{13}$ R. A. Ismail and O. A. Abdulrazaq, Sol. Energy Mater. Sol. Cells 91, 903 (2007).
${ }^{14}$ J. J. Zhu, T. Aaltonen, V. Venkatachalapathy, A. Galeckas, and A. Yu. Kuznetsov, J. Cryst. Growth 310, 5020 (2008).

${ }^{15}$ R. D. Shannon, Acta Crystallogr., Sect. A: Cryst. Phys., Diffr., Theor. Gen. Crystallogr. 32, 751 (1976).

${ }^{16} \mathrm{~J}$. Zuniga-Perez, C. Munuera, C. Ocal, and V. Munoz-Sanjose, J. Cryst. Growth 271, 223 (2004).

${ }^{17}$ U. C. Oh and J. H. Je, J. Appl. Phys. 74, 1692 (1993).

${ }^{18}$ R. Koch, J. Phys.: Condens. Matter 6, 9519 (1994).

${ }^{19}$ J. Pelleg, L. Z. Zevin, and S. Lungo, Thin Solid Films 197, 117 (1991).

${ }^{20}$ J. P. Zhao, X. Wang, Z. Y. Chen, S. Q. Yang, T. S. Shi, and X. H. Liu, J. Phys. D: Appl. Phys. 30, 5 (1997). 